\title{
Neoformação de papila gengival com ácido hialurônico: relato de caso
}

\author{
Neoformation of gingival papilla with hyaluronic acid: a case report
}

Alessandra Kuhn Dall'Magro*
Mônica Dalacort'
Renato dos Santos ${ }^{* * *}$
Rafael Vinícius da Rocha ${ }^{* * * *}$
Túlio del Conde Valcanaiaa
Eduardo Dall'Magro ${ }^{* * * * * *}$

\section{Resumo}

Introdução: a papila interdentária é a porção gengival que ocupa o espaço entre dois dentes adjacentes e a ausência dessa estabelece espaços interdentais denominados black spaces, causando desconforto, impacção alimentar e problemas estéticos e fonéticos. A etiologia da recessão da papila interdental é multifatorial, podendo ou não estar associada às doenças periodontais ou ao tratamento dessas. Objetivo: este estudo tem como objetivo avaliar a eficiência do ácido hialurônico como material indutivo à formação de papila gengival, visando ao preenchimento das ameias interdentais por novo tecido gengival. Relato de caso: paciente branco, 53 anos, gênero masculino, apresentando durante exame clínico perda de papila interdentária na arcada superior, abrangendo os elementos dentários 13, 12, 11, 21, 22 e 23. A saúde bucal dos demais elementos dentários estava em boas condições. Logo após exame clínico e fotografias, foram realizadas aplicações de ácido hialurônico (Perfectha Derm, Obvieline Laboratoire, France) para preenchimento do black space interpapilar. Considerações finais: o ácido hialurônico demonstrou eficácia e biocompatibilidade com os tecidos, obtendo resultados satisfatórios quanto à sua aplicação. A aplicação como material de preenchimento injetável revelou ser de grande importância para casos de grande perda de papila interdentária, demonstrando ser um método simples, seguro e eficaz.

Palavras-chave: Ácido hialurônico. Gengiva. Papila interdentária.

\section{Introdução}

A doença periodontal pode originar perdas da papila interdentária, também designadas de "triângulos negros" ou black spaces, presentes em mais de um terço dos adultos. Além de resultar em imagens sem harmonia estética e causar alterações fonéticas, tais perdas contribuem para a retenção de restos alimentares, afetando a saúde dos tecidos periodontais ${ }^{1}$.

$\mathrm{O}$ ácido hialurônico $(\mathrm{AH})$ é o constituinte mais abundante da matriz extracelular dos tecidos, um glicosaminoglicano não sulfatado de alto peso molecular, com importante papel na hidrodinâmica tecidual e na função anti-inflamatória ${ }^{2}$. Recentemente, esse biomaterial tem sido sugerido na reconstrução das papilas interdentárias e dos colarinhos metálicos, demonstrando resultados eficientes ${ }^{2}$.

A papila interdental é classificada como tecido gengival não queratinizado ou paraqueratinizado, recoberto por epitélio escamoso estratificado. $\mathrm{Na}$ região anterior, apresenta formato piramidal e ocupa o espaço localizado entre dois dentes adjacentes, coronalmente à crista óssea alveolar, abaixo do ponto de contato. Na região posterior, a papila é ampla e apresenta uma área côncava não queratinizada, denominada área do "col", que une a papila vestibular à lingual. É uma estrutura que tem suprimento vas-

Especialista em Cirurgia e Traumatologia Bucomaxilofacial (CTBMF), Santa Casa, Porto Alegre. Mestre em Ciências Médicas pela Faculdade de Medicina da Universidade Federal do Rio Grande do Sul. Professora dos cursos de Pós-Graduação em CTBMF do Centro de Estudos Odontológicos Meridional (Ceom), Passo Fundo, RS, e do Instituto Odontológico das Américas (IOA), Balneário Camboriú, SC. Membro do Corpo Clínico do Hospital São Vicente de Paulo de Passo Fundo (HSVP) e do Instituto de Ortopedia e Traumatologia (IOT), Passo Fundo, RS, Brasil.

Cirurgiã-dentista, Faculdade de Odontologia da Universidade de Passo Fundo (FO-UPF), Passo Fundo, RS, Brasil.

Especialista em CTBMF, Ceom, HSVP, Passo Fundo, RS, Brasil.

Mestre em Clínica Odontológica pela FO-UPF. Professor titular da FO-ITPAC Porto Nacional, TO, Brasil.

Especialista e mestre em CTBMF, Universidade Federal de Pelotas. Doutor em CTBMF, Pontifícia Universidade Católica do Rio Grande do Sul. Professor de CTBMF da Faculdade Avantis. Coordenador do curso de especialização em Implantodontia da Faculdade Avantis, Balneário Camboriú, SC, Brasil.

****** Especialista em Prótese Dentária e mestre em Laser em Odontologia, Universidade de São Paulo. Mestre e doutor em Materiais Dentários, Unicamp. Professor titular III da FO-UPF, Passo Fundo, RS, Brasil. 
cular sanguíneo mínimo, o que dificulta sua reconstrução ${ }^{3}$. A ausência dessa estrutura torna as ameias gengivais abertas, podendo desencadear problemas fonéticos, impacção alimentar e desconforto estético $^{3}$. O aumento gengival, a pouca espessura de rebordo, a recessão gengival e, principalmente, a ausência de papila têm sido as maiores causas da perda da harmonia da gengiva com os dentes naturais e com os reconstruídos ${ }^{4}$.

A recessão periodontal apresenta etiologia multifatorial, com a combinação de variáveis externas e anatômicas. As características anatômicas que podem estar associadas ao posicionamento dentário são constituídas pelas dimensões ósseas e mucogengivais locais, sendo a espessura da gengiva marginal de extrema importância. Já existem várias pesquisas mostrando que a melhor prevenção para uma doença da cavidade oral é a correta higienização $0^{5}$.

Existem diversos materiais injetáveis para aumento de partes moles. Um implante ideal, além de ser biocompatível, não deve ser pirogênico, não deve causar processo inflamatório, e deve ser atóxico, de fácil uso e estável após aplicação, não ser migratório, ter longa duração e aparência natural, ser clínica e quimicamente inerte e não ser carcinogênico ou teratogênico ${ }^{4}$. Os agentes de preenchimento dérmico de uso corrente se dividem em quatro categorias: xenoenxertos (ácido hialurônico), autoenxertos, homoenxertos e de materiais sintéticos. Apesar dos avanços na tecnologia e da existência de vários biomateriais de uso corrente no mercado, nenhum deles alcança todos os critérios assinalados ${ }^{6}$.

$\mathrm{O}$ AH representa uma alternativa no tratamento do envelhecimento facial e tem sido utilizado há mais de uma década no preenchimento de partes moles para corrigir depressões, rugas e sulcos. Suas potenciais complicações são raras, autolimitadas e incluem reações inflamatórias, pequenos hematomas e/ou equimoses, abscessos nos sítios de aplicação, necrose tecidual (por injeção intravascular ou compressão da rede vascular adjacente), edema persistente e granulomas ${ }^{7}$.

A substância absorvível de maior experiência na prática clínica é o AH. Há diversas apresentações no mercado e a durabilidade dos efeitos clínicos tem variado, sem trabalhos científicos comparativos. Por sua alta biocompatibilidade e ausência de reação imunológica local ou sistêmica, permite eliminar o teste cutâneo preliminar, apresentando amostras livres de fibrose e de reação de corpo estranho importante $^{6}$. O objetivo deste trabalho é avaliar a eficiência do $\mathrm{AH}$ como material indutivo à formação de papila gengival, visando ao preenchimento das ameias interdentais.

\section{Relato de caso}

Paciente branco, 53 anos de idade, do gênero masculino, procurou atendimento com o intuito de submeter-se a um tratamento inovador na área de enxertos injetáveis. Durante exame clínico, foi observada a presença de perda de papila interdentária na arcada superior, abrangendo os elementos dentários 13, 12, 11, 21, 22 e 23 (Figura 1a, 1b, 1c) originando os chamados black spaces. A saúde bucal dos demais elementos dentários estavam em boas condições. Logo após exame clínico, preenchimento do termo de consentimento informado e esclarecido e realização de fotografias, foram realizadas as primeiras aplicações do material de preenchimento injetável escolhido, o AH (Perfectha Derm, Laboratoire Obvieline, France).

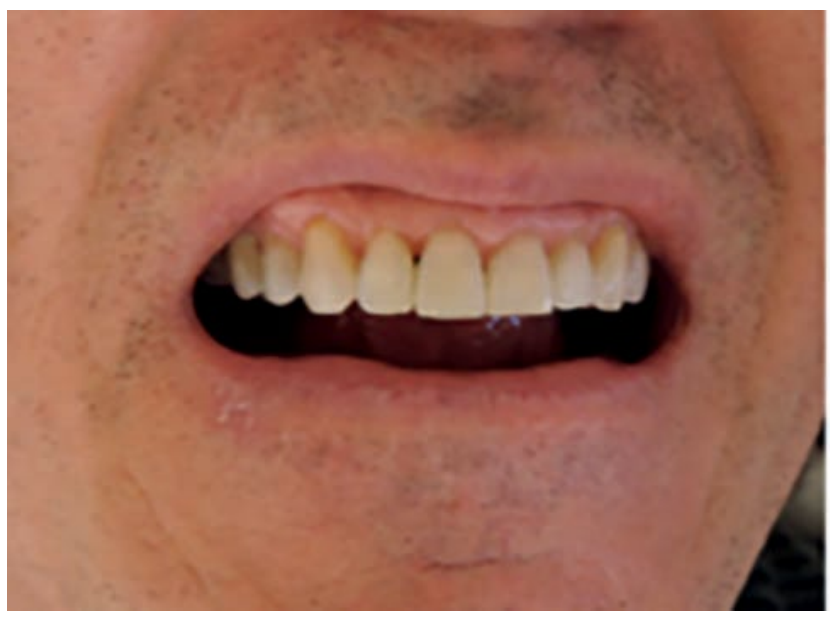

Figura 1a - Situação inicial mostrando perda de papila interdentária na região entre os elementos 11 e 12

Fonte: elaboração dos autores.

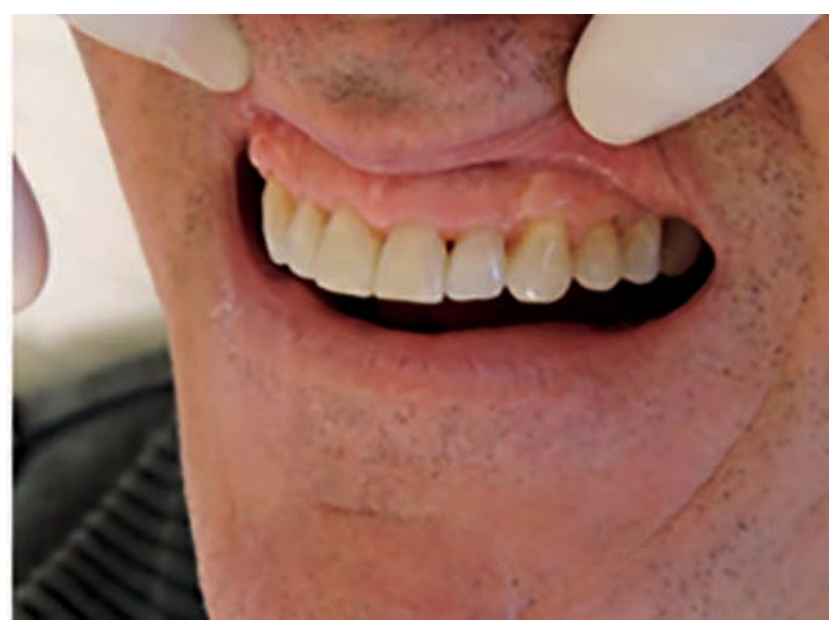

Figura 1 - Situação inicial mostrando perda de papila interdentária na região entre os elementos 21 e 22

Fonte: elaboração dos autores. 


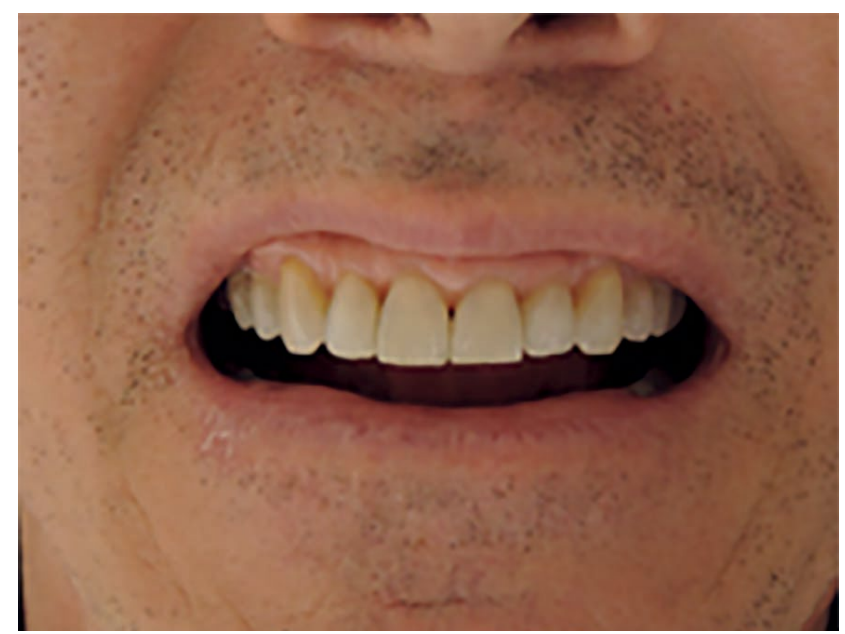

Figura 1c - Situação inicial mostrando perda de papila interdentária na região dos dentes 11 e 21

Fonte: elaboração dos autores.

O paciente foi previamente anestesiado com anestesias infiltrativas em fundo de sulco anterior da maxila (do 13 ao 23) para posterior aplicação de uma quantidade de meia seringa do produto dividido em 5 partes (0,1 ml em cada papila) (Figuras 2 a 4$)$.

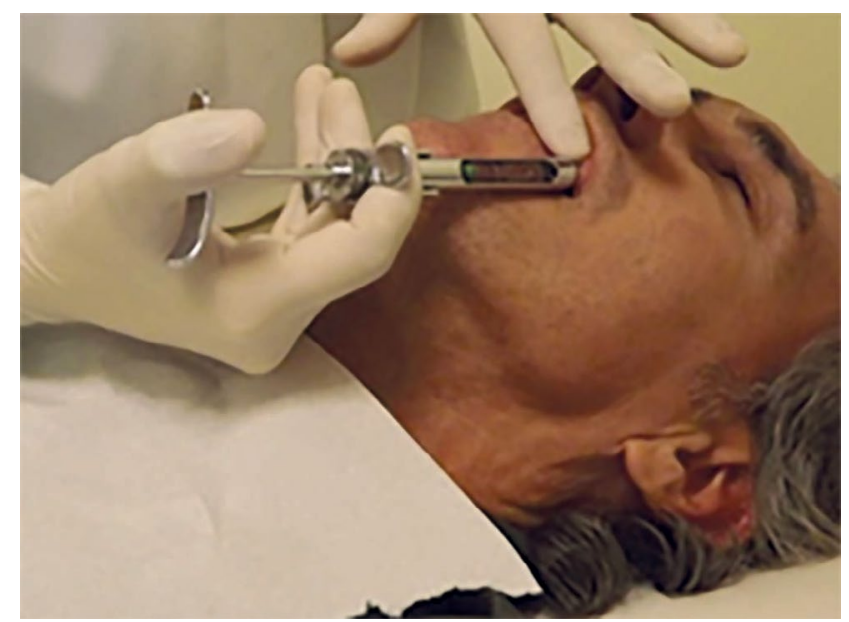

Figura 2 - Paciente sendo anestesiado

Fonte: elaboração dos autores.

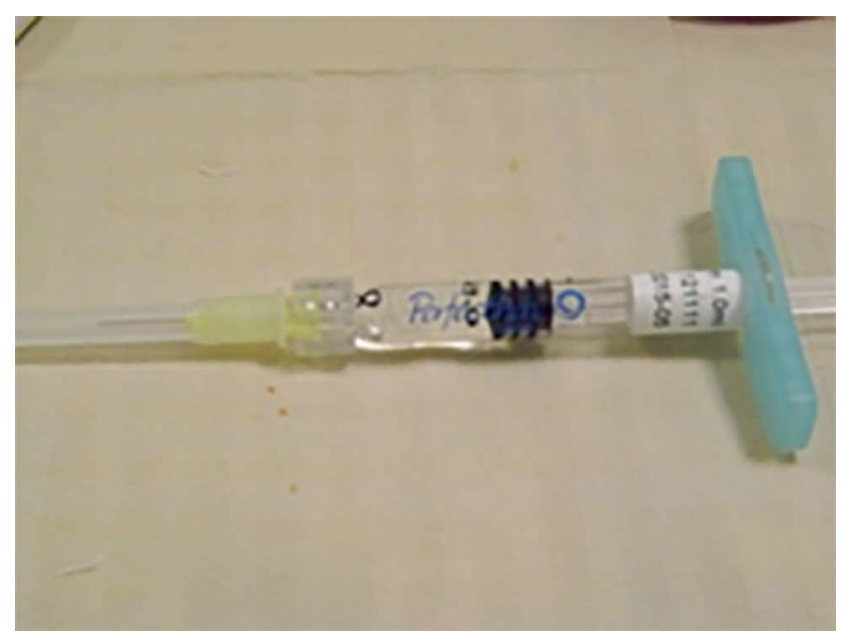

Figura 3 - Seringa com AH Perfectha

Fonte: elaboração dos autores.

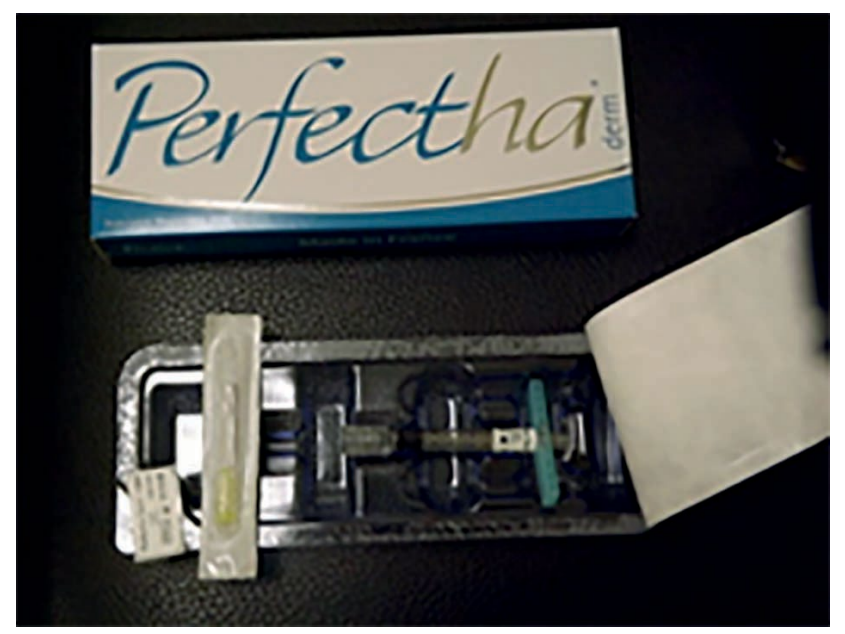

Figura 4 - Kit Perfectha Derm contendo uma seringa com $1 \mathrm{ml}$ do produto e agulha mostrando adesivo com data de fabricação, validade e número do lote (rastreabilidade)

Fonte: elaboração dos autores.

Decorridos trinta dias da primeira aplicação, o paciente retornou para a segunda aplicação. A quantidade aplicada foi a mesma utilizada no primeiro procedimento (Figuras 5 a 7 ).

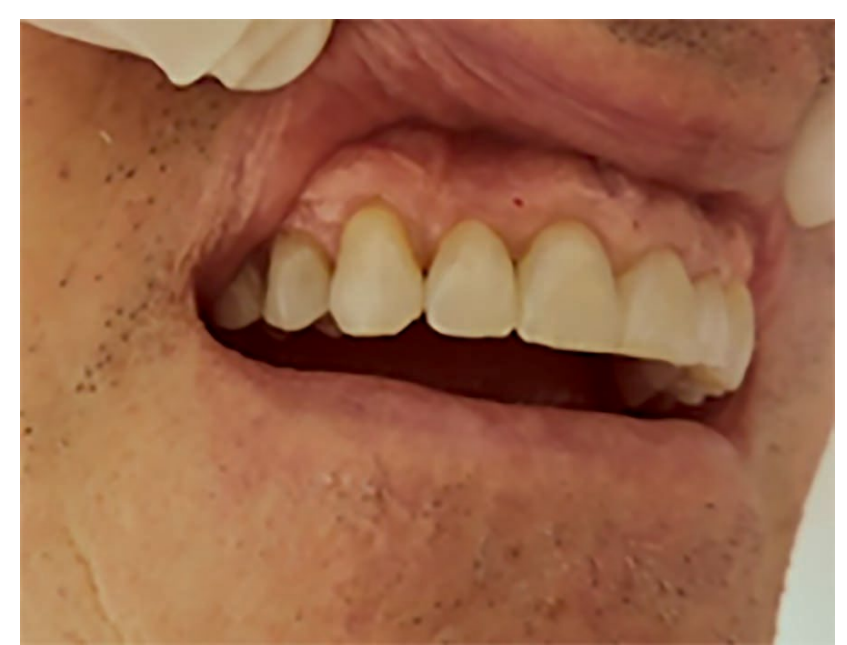

Figura 5 - Aplicação e resultado final

Fonte: elaboração dos autores.

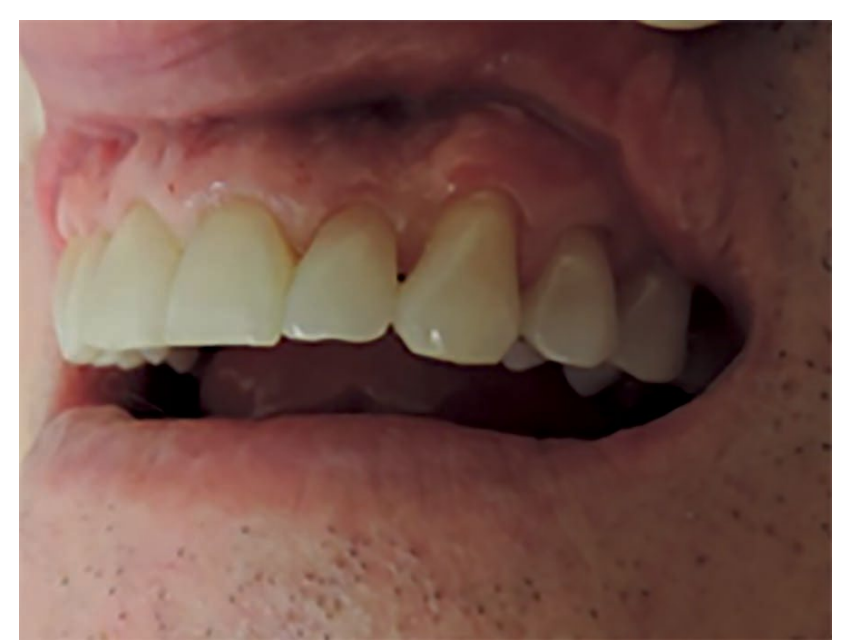

Figura 6 - Aplicação e resultado final

Fonte: elaboração dos autores. 


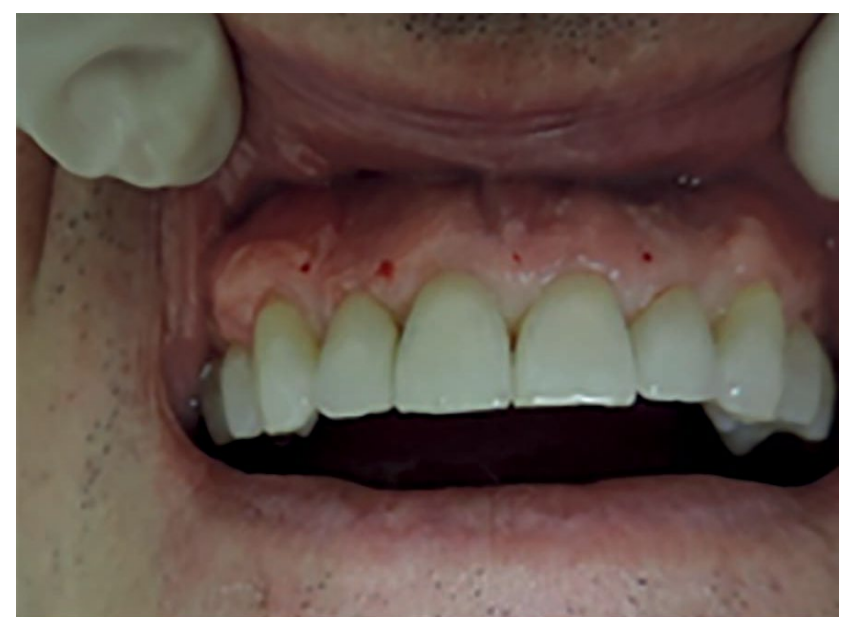

Figura 7 - Aplicação e resultado final

Fonte: elaboração dos autores.

\section{Discussão}

O equilíbrio da face e do sorriso é a busca incessante da odontologia atual. A procura por dentes cada vez mais brancos, alinhamentos mais corretos e colocação de próteses em espaços desdentados faz com que a opção de implantes dentários e a enxertia de materiais injetáveis de preenchimento, entre outros, sejam as novas tendências da Odontologia Moderna.

Existem vários materiais injetáveis de preenchimento que podem ser usados para diversas finalidades. Para área estética, na região de perdas de papila interdentais, está sendo usado o $\mathrm{AH}$, que é uma substância produzida em nosso organismo, um líquido altamente solúvel, sendo encontrado em maior parte na nossa pele, que confere ao órgão volume, sustentação, hidratação e elasticidade. Seu uso na região de papilas interdentais perdidas tem mostrado excelentes resultados, com melhor harmonia do sorriso e completa integração aos tecidos.

A imitação dos dentes naturais, tanto na sua função quanto em sua estética, tem chegado a um nível muito alto, porém, os tecidos moles que os envolvem, dependendo do caso, podem prejudicar essa estética. Devido à pobre vascularização das papilas dentárias e à quantidade de perda óssea, muitas técnicas cirúrgicas para a reconstrução papilar apresentam resultado limitado ${ }^{4}$.

Em estudos utilizando o $\mathrm{AH}$ a $3 \%$ no preenchimento de sulco nasogeniano (SNG), foi constatado um aumento da espessura cutânea até 12 meses, seguido de um decréscimo progressivo ${ }^{7}$. Por esse motivo, após um ano, o paciente que recebe o preenchimento papilar precisa retornar para avaliação e possíveis técnicas incrementais poderão ser sugeridas.

Em estudo realizado com o produto Perfectha ${ }^{\circledR}$, preenchedor à base de $\mathrm{AH}$, para correção dos sulcos nasolabiais e contorno dos lábios ${ }^{6}$, mesmo produto utilizado neste caso clínico, os autores observaram, uma semana após a aplicação do preenchedor, uma melhora nos sulcos nasolabiais de $86 \%$ das mulheres e nos lábios de $89 \%$ delas. Reação inflamatória transitória, leve ou moderada, e equimoses ocorreram em $15 \%$ e $9 \%$ das pacientes, respectivamente, sobretudo nos sulcos nasolabiais. Os bons resultados se mantiveram em $76 \%$ e $57 \%$ das mulheres, nos sulcos nasolabiais, e em $72 \%$ e $45 \%$ delas, nos lábios, após 3 e 6 meses, respectivamente. Assim, concluíram que o preenchedor Perfectha ${ }^{\circledR}$ mostrou-se eficaz e seguro para essas indicações ${ }^{8}$, assim como evidenciou-se como indicado para o preenchimento de black spaces realizado neste trabalho.

A eficácia da aplicação de gel de AH para preenchimento da papila interdentária foi verificada em estudo realizado por Lima et al. (2012) ${ }^{9}$. Foram escolhidas áreas teste e áreas controle em cinco pacientes. A área teste foi submetida à injeção de gel de AH $2 \mathrm{~mm}$ a $3 \mathrm{~mm}$ por apical da extremidade da papila (menos de $0,2 \mathrm{ml}$ ), e na área controle foi injetada uma pequena quantidade de anestésico local. Foi repetida a intervenção até três vezes, com três semanas de intervalo entre cada uma. A avaliação do preenchimento foi realizada por meio de fotografias iniciais e finais e análise com o programa GIMP 2 para comparação das diferenças entre essas. Os resultados mostraram que duas áreas teste obtiveram preenchimento de $100 \%$ e três obtiveram preenchimento entre $38,45 \%$ e $58,5 \%$. As áreas controle sofreram variações entre $16,7 \%$ e $9,5 \%$. $\mathrm{O}$ aumento foi estatisticamente significativo com a aplicação do produto $(\mathrm{p}=0,002)$. Os autores concluíram que a aplicação de AH parece ser uma alternativa válida no preenchimento da papila interdentária, o que está em conformidade com nosso trabalho, em que foi observado, também por meio de fotografias, o preenchimento semelhante ao citado.

Francischone $^{10}(2011)$ avaliou a manutenção dos níveis (altura e áreas) papilares em regiões estéticas que receberam um implante, dois implantes contíguos, ou dois implantes contíguos associados a enxertos de tecido ósseo e tecido gengival. A amostra contou com trinta pacientes, de ambos os gêneros, que apresentavam duas ausências dentárias contíguas em área estética da maxila. $\mathrm{O}$ autor concluiu que as alturas e áreas papilares sofreram maiores perdas com dois implantes contíguos e, ainda, que, para a instalação de dois implantes contíguos em área estética, recomenda-se a utilização prévia de enxertos ósseos associados a enxertos de tecido gengival. Também Bhansali et al. ${ }^{11}$ (2010) realizaram outra técnica para preservar a estética entre dentes com raízes naturais, permitindo melhor perfil de emergência e evitando o estrangulamento da papila interdental e, por consequência, a formação de black spaces ${ }^{11}$.

Sawai e Kohad ${ }^{12}$ (2012) reconstruíram cirurgicamente papilas interdentais com tecido gengival para finalidade estética e manutenção da saúde bucal em pacientes com queixa de black spaces na região anterior da maxila. Os autores relataram que 
a técnica cirúrgica utilizada para a reconstrução da papila interdental teve bastante sucesso e que a utilização de enxertos ósseos ou de tecidos moles dos enxertos teriam melhorado ainda mais os resultados. Essa informação concorda com o estudo de Oliveira et al..$^{13}$ (2012), que estabelece o tratamento cirúrgico e não cirúrgico para recuperação da papila interdental e demonstra que se a distância da crista ao ponto de contato for igual ou menor que $5 \mathrm{~mm}$, a papila estará presente em quase $100 \%$ dos casos; e se a distância for maior que $5 \mathrm{~mm}$, haverá pouca papila na maioria dos casos. Neste caso clínico, foram testadas soluções menos invasivas para solucionar o problema de paciente com black spaces.

Em um estudo ${ }^{14}$, a eficácia do preenchimento dos sulcos nasojugal e palpebromalar foi verifica$\mathrm{da}^{14}$ por meio da injeção de AH. Também, foram analisadas as possíveis complicações, utilizando dez pacientes de ambos os sexos, com idades entre 25 e 60 anos, os quais apresentavam sulcos nasojugal e palpebromalar deprimidos e que não haviam sido submetidos à cirurgia na pálpebra inferior nem apresentavam histórico de trauma nesse local. O AH foi aplicado via transcutânea e depositado na região supraperiosteal em pequenos volumes. Todos os pacientes foram tratados pelo mesmo cirurgião, examinados no pós-operatório em intervalos regulares e fotografados no pré-operatório, no pós-operatório imediato, na $4 .^{\mathrm{a}}$ semana, no $6 .^{\circ}$ e no $12 .^{\circ}$ mês após o procedimento. As complicações observadas foram: equimose em seis casos, edema local por mais de 48 horas em um paciente e lesão cutânea semelhante à acne em outro paciente. Este estudo demonstrou que o tratamento dos sulcos nasojugal e palpebromalar, com uso de AH se mostrou eficaz e previsível, com alto nível de satisfação dos pacientes e com resultado duradouro ${ }^{14}$, o que também foi observado no caso clínico deste trabalho, porém sem as complicações observadas no trabalho supracitado.

Braz e Mukamal ${ }^{15}$ (2011) realizaram estudo sobre a técnica de preenchimento labial com AH, utilizando microcânulas para o tratamento de três diferentes características labiais, alcançando resultados distintos: definição do contorno, projeção e aumento do volume dos lábios. Foram tratados 55 pacientes, sendo 47 mulheres e 8 homens, com idades entre 18 e 71 anos. Os pacientes relataram alto grau de satisfação, e os autores concluíram que com microcânulas é seguro trabalhar em plano profundo e subdérmico, reduzindo os riscos, desde que o procedimento seja realizado com habilidade e delicadeza.

\section{Considerações finais}

A indicação do AH para neoformação papilar demonstrou ser um método simples, seguro, eficaz e minimamente invasivo, como ilustrado no caso clínico deste trabalho.

\section{Abstract}

Introduction: Interdental papilla is the gingival portion that occupies the space between two adjacent teeth, and the absence of this structure establishes interdental spaces called black spaces, which cause discomfort, food impaction, and phonetic and aesthetic problems. The etiology of interdental papilla recession is multifactorial and may or may not be associated with periodontal diseases or their treatment. Objective: The objective of this study is to assess the efficiency of hyaluronic acid as inductive material to the formation of gingival papilla, aiming to fill interdental niches with new gingival tissue. Case report: Male Caucasian patient, 53 years old, whose clinical examination presented loss of interdental papilla in the upper arch, involving dental elements 13, 12, 11, 21, 22, and 23. The oral health of the remaining dental elements was good. Right after clinical examination and photographs, hyaluronic acid (Perfectha Derm - Derm Brand - Made in France) was applied to fill the interpapillary black space. Final considerations: Hyaluronic acid showed effectiveness and biocompatibility with tissues in their various studies, obtaining satisfactory results for its application. Injectable filling material application was found to be very important in cases of great loss of interdental papilla, proving to be a simple, safe, and effective method.

Keywords: Gingiva. Hyaluronic acid. Interdental papilla.

\section{Referências}

1. Celória A. Eficiência do ácido hialurônico na regeneração das papilas interdentárias e dos colarinhos metálicos em implantes dentários. In: Cauduri R. Toxina botulínica \& preenchedores na odontologia. Porto Alegre: RGO; 2015. p. 112-21.

2. Antonio CR. Tratamento da hiperpigmentação periorbital com preenchimento de ácido hialurônico justa ósseo através de cânula: uma avaliação retrospectiva. RBM, Especial Dermatologia 2012; 1:24-9.

3. Kovalik AC, Bonafé ETR, Pilatti GL, Santos FA. Papila interdental: previsibilidade das técnicas reconstrutivas. Braz J Periodontol 2011; 21(3):22-32.

4. Cavalcanti SM. Reconstrução cirúrgica de papilas interdentais [Monografia de Especialização em Periodontia]. Piracicaba: Faculdade de Odontologia de Piracicaba, Universidade Estadual de Campinas; 2002.

5. Yared KFG, Zenobio EG, Pacheco W. A etiologia multifatorial da recessão periodontal. Rev Dent Press Ortodon Facial 2006; 11(6):45-51.

6. Rosa SC, Macedo LS. Materiais injetáveis para aumento de partes moles. Rev Soc Bras Cir Plást 2007; 22(2):116-21.

7. Salles AG, Remigio AFN, Zacchi VBL, Salto OC, Ferreira MC. Avaliação clínica e da espessura cutânea um ano após preenchimento de ácido hialurônico. Rev Bras Cir Plást (Impr.) 2011; 26(1):257-60.

8. Talarico S, Hassun MK, Monteiro E, Parada MO, Buratini B L, Arruda L, et al. Avaliação da segurança e eficácia de novo preenchedor a base de ácido hialurônico no tratamento de sulcos nasolabiais e contorno dos lábios. Surg Cosmet Dermatol 2010; 2(2):83-6. 
9. Lima DI, Pinto M, Mendes L. Eficácia do ácido hialurônico no preenchimento da papila interdentária. Congresso OMD, 2012. [periódico on-line]. [citado 2013 nov. 13]. Disponível em URL: http://www.omd.pt/congresso/2012/apresentacoes/ p153.

10. Francischone CA. Estudo das dimensões da papila gengival peri e interimplantar através da gengimorfometria [Tese de Doutorado em Implantodontia]. Bauru: Universidade Sagrado Coração; 2011.

11. Bhansali S, Bhansali N, Singh K, Agarwal B. Uma técnica de impressão para preservar papila interdental. J Indiano Prosthodont Soc 2010; 10(4):226-9.

12. Sawai ML, Kohad RM. Uma avaliação de um procedimento cirúrgico plástico periodontal para a reconstrução da papila interdental na região anterior da maxila: um estudo clínico. J Indiano Soc Periodontol 2012; 16(4):533-8.

13. Oliveira DJ, Storrez MC, Souza MA, Lopes RT, Vieira SJ, Deliberador MT. Papillary regeneration: anatomical aspects and treatment approaches. RSBO 2012; 9(4):448-56.

14. Viana GAP, Osaki MH, Cariello AJ, Damasceno RW. Tratamento dos sulcos palpebromalar e nasojugal com ácido hialurônico. Arq Bras Dental 2011; 74(1):44-47.

15. Braz AV, Mukamal LV. Preenchimento labial com microcânulas. Surg Cosmet Dermatol 2011; 3(3):257-60.

\section{Endereço para correspondência:}

Alessandra Kuhn Dall'Magro

Rua Teixeira Soares 777/02

Ed. Columbia Work Center

99010-080 Passo Fundo, RS

Telefone: (54) 81119877

E-mail: alessandrakuhn@hotmail.com

Recebido: 14/04/2015. Aceito: 25/05/2016. 